\title{
ON THE SPECTRUM OF MONOIDS AND SEMILATTICES
}

\author{
ILIA PIRASHVILI
}

\section{INTRODUCTION}

In the last years there was considerable interest in the spectrum of commutative monoids [9], [1], [6, [7], [2], [3], [5], [4]. These objects play the same role in the theory of schemes over the 'field with one element' as the spectrum of rings played in the theory of schemes over rings. See also [10] for more about geometry over the field with one element.

The aim of this short note is to prove a useful lemma on the spectrum of commutative monoids and some of its immediate consequences. Our lemma claims that for any commutative monoid $M$ one has a natural isomorphism

$$
\operatorname{Spec}(M) \cong \operatorname{Hom}(M, \mathbb{I}),
$$

where Hom is taken in the category of commutative monoids and $\mathbb{I}=\operatorname{Spec}(\mathbb{N})$. The monoid structure in the spectrum of a monoid, is given by the union of prime ideals. From isomorphism (1), one easily deduces the 'reduction isomorphism'

$$
\operatorname{Spec}(M) \cong \operatorname{Spec}\left(M^{s l}\right),
$$

where $M^{s l}$ is $M$ modulo to the relation $a^{2}=a$. Thus the study of the spectrum of commutative monoids, reduces to the study of the spectrum of semilattices. Our second main result claims that if $L$ is a semilattice, then there is an injective map

$$
\alpha_{L}: L \rightarrow \operatorname{Spec}(L),
$$

which is bijective provided $L$ is finitely generated (hence finite). In particular these results give an effective way of computing $\operatorname{Spec}(M)$ for an arbitrary finitely generated commutative monoid $M$. We also show that $\alpha$ is "natural" in the following sense. If $f: L \rightarrow L^{\prime}$ is a morphism of finitely generated semilattices, then one has the following commutative diagram

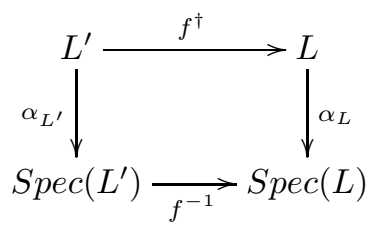

where $f^{\dagger}$ denotes the right adjoint of $f$. The case of infinite semilatices is also considered.

Most of the results in this note were part of my 4th year masters thesis at UCL, under the supervision of Dr. Javier López Peña, who has introduced me to this subject. But Isomorphism (1), which is new, not only simplifies much, but also gives a new insight in the spectrum of monoids.

\section{Prime ideals of commutative monoids}

In what follows all monoids are commutative and they are written multiplicatively. Let $M$ be a monoid, then a subset $\mathfrak{a}$ is called an ideal provided for any $a \in \mathfrak{a}$ and $x \in M$ one has $a x \in \mathfrak{a}$. For an element $a \in M$, we let $(a)$ be the principal ideal $a M$. An ideal $\mathfrak{p}$ is called prime, provided $\mathfrak{p} \neq M$ and the complement $\mathfrak{p}^{c}$ of $\mathfrak{p}$ in $M$ 
is a submonoid. Thus an ideal $\mathfrak{p}$ is prime iff $1 \notin \mathfrak{p}$ and if $x y \in \mathfrak{p}$ then either $x \in \mathfrak{p}$ or $y \in \mathfrak{p}$. We let $\operatorname{Spec}(M)$ be the set of all prime ideals of $M$. It is equipped with a topology, where the sets

$$
D(a)=\{\mathfrak{p} \in \operatorname{Spec}(M) \mid a \notin \mathfrak{p}\}
$$

form a bases of open sets. Here $a$ is an arbitrary element of $M$, see [9, [6, [7]. Observe that if $f: M_{1} \rightarrow M_{2}$ is a homomorphism of monoids then for any prime ideal $\mathfrak{q} \in \operatorname{Spec}\left(M_{2}\right)$ the pre-image $f^{-1}(\mathfrak{q})$ is a prime ideal of $M_{1}$, hence any monoid homomorphism $f: M_{1} \rightarrow M_{2}$ gives rise to a continuous map

$$
f^{*}: \operatorname{Spec}\left(M_{2}\right) \rightarrow \operatorname{Spec}\left(M_{1}\right) ; \quad \mathfrak{q} \mapsto f^{-1}(\mathfrak{q}) .
$$

It is obvious that the union of prime ideals is again a prime ideal. It is also clear that the empty set is a prime ideal, which is the least prime ideal, and the set of noninvertible elements of $M$ is a prime ideal, which is the greatest prime ideal. Thus $\operatorname{Spec}(M)$ is a (topological) monoid with respect to union.

Let $\mathbb{I}=\{0,1\}$ be the monoid with the obvious multiplication. We will equip the set $\mathbb{I}$ with the topology, where the subsets $\emptyset,\{1\}, \mathbb{I}$ are all open sets. Let $\mathbf{N}=$ $\left\{1, t, t^{2}, \cdots\right\}$ be the free monoid with one generator (of course it is isomorphic to the additive monoid of natural numbers). Then $\operatorname{Spec}(\mathbf{N})=\{\emptyset,(t)\}$. Observe that $\emptyset$ is an open subset of $\operatorname{Spec}(\mathbf{N})$. Hence $\mathbb{I} \cong \operatorname{Spec}(\mathbf{N})$ as topogical monoids.

Lemma 2.1. For any commutative monoid $M$ one has a natural isomorphism of topological monoids

$$
\operatorname{Spec}(M) \cong \operatorname{Hom}(M, \mathbb{I}),
$$

where the topology on the right hand side is induced by the the product topology on $\prod_{m \in M} \mathbb{I}$.

Proof. Observe that $\{0\}$ is a prime ideal of $\mathbb{I}$ (in fact $\operatorname{Spec}(\mathbb{I})=\{\emptyset,\{0\}\} \cong \mathbb{I}$ ). Hence for any homomorphism $f: M \rightarrow \mathbb{I}$ we have $f^{-1}(0) \in \operatorname{Spec}(M)$. In this way we obtain a map $\theta: \operatorname{Hom}(M, \mathbb{I}) \rightarrow \operatorname{Spec}(M)$. The inverse of this map is given as follows, for a prime ideal $\mathfrak{p} \in \operatorname{Spec}(M)$, we let $f_{\mathfrak{p}}: M \rightarrow \mathbb{I}$ be the map given by

$$
f_{\mathfrak{p}}(x)= \begin{cases}0, & \text { if } x \in \mathfrak{p} \\ 1, & \text { if } x \notin \mathfrak{p}\end{cases}
$$

Now it is easy to check that $f_{\mathfrak{p}}: M \rightarrow \mathbb{I}$ is a homomorphism. Hence $\mathfrak{p} \mapsto f_{\mathfrak{p}}$ defines a map $\operatorname{Spec}(M) \rightarrow \operatorname{Hom}(M, \mathbb{I})$ which is clearly a homomorphism and is the inverse of $\theta$. It is also trivial to check that $\theta$ and its inverse are continuous.

Since $\operatorname{Hom}(-, \mathbb{I})$ takes colimits to limits we have (compare pp. 5 and 6 in [4]).

Corollary 2.2. If $J$ is a poset and $\left(M_{j}\right)_{j \in J}$ is a direct system of monoids indexed by $J$, then the natural map

$$
\operatorname{Spec}\left(\operatorname{colim}_{j \in J} M_{j}\right) \rightarrow \lim _{j \in J} \operatorname{Spec}\left(M_{J}\right)
$$

is an isomorphism.

Recall that the category of monoids satisfying the identity $a^{2}=a$ is equivalent to the category of join semilattices 8 . By a join semilattice we mean a poset $L$ with a least element such that for any two elements $a, b \in L$ there exist the join $a \vee b$, which is the least element among the elements $x$ such that $a \leq x$ and $b \leq x$. If $M$ is a monoid such that $m^{2}=m$ holds for all $m \in M$, then one defines $x \leq y$ if $x y=y$. It is easily seen that in this way we obtain a join semilattice. Conversely, if $L$ is a join semilattice we can consider $L$ as a monoid, with operation

$$
x y:=x \vee y .
$$

The least element of $L$ is the unit in this monoid. It is clear that we have $x^{2}=x$ for all $x \in L$. 
We let SL be the full subcategory of monoids $M$ satisfying the identity $x^{2}=x$ for all $x$. For example we have $\mathbb{I} \in \mathbf{S L}$. It is well-known (and trivial) that the inclusion SL $\subset$ Mon has a left adjoint functor $M \mapsto M^{s l}$, where $M^{s l}$ is the quotient of $M$ by the smallest congruence $\sim$ for which $x \sim x^{2}$ for any $x \in M$. For example $\mathbb{I}=(\mathbb{N})^{s l}$. For the reader familiar to the tensor product of commutative monoids we also mention the isomorphism $M^{s l} \cong M \otimes \mathbb{I}$. However we will not make use of this fact. As it follows from Lemma 2.1 and Corollary 3.7 below, the object II is a dualizing object in the category of finite monoids satisfying the identity $m^{2}=m$, that is for any such $M$ the following map is an isomorphism

$$
e v: M \cong \operatorname{Hom}(\operatorname{Hom}(M, \mathbb{I}), \mathbb{I}),
$$

where $(e v(m))(f)=f(m), m \in M, f \in \operatorname{Hom}(M, \mathbb{I})$.

Lemma 2.3. (Reduction lemma). For any monoid $M$, the canonical homomorphism $q: M \rightarrow M^{\text {sl }}$ yields the isomorphism

$$
q^{-1}: \operatorname{Spec}\left(M^{s l}\right) \rightarrow \operatorname{Spec}(M) .
$$

Proof. By definition of $M^{s l}$ for any $X \in \mathbf{S L}$ one has $\operatorname{Hom}(M, X)=\operatorname{Hom}\left(M^{s l}, X\right)$. By putting $X=\mathbb{I}$ the result follows from Lemma 2.1.

As a consequence we obtain the following fact which sharpens Lemma 4.2 in [7]. We will make use of the following description of $M^{s l}$. Thanks to Theorem 1.2 of Chapter III in [8]) one has $M^{s l}=M / \sim$ where $a \sim b$ provided there exist natural numbers $m, n \geq 1$ and elements $u, v \in M$ such that $a^{m}=u b$ and $b^{n}=v a$.

Corollary 2.4. Let $B$ a submonoid of $A$ and assume for any element $a \in A$ there exist a natural number $n$ such that $a^{n} \in B$. Then

$$
\operatorname{Spec}(A) \rightarrow \operatorname{Spec}(B)
$$

is an isomorphism.

Proof. It suffice to show that $B^{s l} \rightarrow A^{s l}$ is an isomorphism. Take any element $a \in A$, since $a \sim a^{n}$ for all $n$ we see that the map in question is surjective. Now take two elements $b_{1}, b_{2}$ in $B$ and assume $b_{1} \sim b_{2}$ in $A$. Then there are $u, v \in A$ such that $b_{1}^{k}=u b_{2}$ and $b_{2}^{m}=v b_{1}$. Take $N$ such that $u_{1}=u^{N} \in B$ and $v_{1}=v^{N} \in B$. Then $b_{1}^{k N}=u_{1} b_{2}^{N}$ and $b_{2}^{N}=v_{1} b_{1}^{N}$. Thus $b_{1} \sim b_{2}$ in $B$ and we are done.

\section{SpeCtrum OF SEMILATtices}

By the reduction lemma 2.3 the study of the spectrum of commutative monoids, reduces to the study of the spectrum of semilattices. Before we go further let us fix some terminology.

3.1. Adjoint maps of morphisms of semilattices. The main result of this section is probably well-known to experts, but I couldn't find any references.

For a poset $P$ we let $P^{o p}$ be the poset, which is $P$ as a set, but with the reverse ordering. We sometimes use the notations

$$
\operatorname{Max}\{x \mid x \in P\} \text { and } \operatorname{Min}\{x \mid x \in P\}
$$

for the greatest and least elements of $P$.

Let $X$ and $Y$ be posets. Assume $f: X \rightarrow Y$ and $g: Y \rightarrow X$ be maps. We say that $g$ is a left adjoint of $f$ and $f$ is a right adjoint of $g$, if

$$
(y \leq f(x)) \Leftrightarrow(g(y) \leq x)), \quad x \in X, y \in Y .
$$

As a specialization of the well-known facts from the category theory we can conclude that if $f$ has a left or right adjoint then it is unique. We sometimes write $g=f^{*}$ for the left adjoint of $f$ and $f=g^{\dagger}$ for the right adjoint of $g$.

Proposition 3.1. Let $X$ and $Y$ be posets. 
(1) A map $f: X \rightarrow Y$ has a right adjoint $f^{*}$ if and only if for any $y \in Y$ the set $\{x \in X \mid y \leq f(x)\}$ has the least element. If this is the case, then

$$
f^{*}(y)=\operatorname{Min}\{x \in X \mid y \leq f(x)\} .
$$

If this is the case, then both $f$ and $g$ are monotonic maps.

(2) A map $f: X \rightarrow Y$ has a left adjoint $f^{\dagger}: Y \rightarrow X$ if and only if for any $y \in Y$ the set $\{x \in X \mid f(x) \leq y\}$ has the greatest element. If this is the case, then

$$
f^{*}(y)=\operatorname{Max}\{x \in X \mid f(x) \leq y\} .
$$

If this is the case, then both $f$ and $g$ are monotonic maps.

A lattice is a poset $L$ which is simultaneously a join and meet semi-lattice (that is the opposite poset is a join semi-lattice).

Lemma 3.2. If $L$ is a finite join semi-lattice, then $L$ is a lattice.

Proof. First we show that $L$ posses the greatest element. In fact, if $L=\left\{x_{1}, \cdots, x_{n}\right\}$, then $x_{1} \vee \cdots \vee x_{n}$ is the greatest element. Now for any $a, b \in L$ we consider the subset

$$
Q_{a, b}:=\{x \in L \mid x \leq a, \text { and } x \leq b\} .
$$

It is clear that the least element belongs to $Q_{a, b}$, so it is nonempty. It is also clear that $Q_{a, b}$ is a join subsemilattice of $L$. Thus $Q_{a, b}$ has the greatest element $a \wedge b$ and we are done.

If $f: L \rightarrow L^{\prime}$ is a morphism of finite join semilattices then as we have seen $L$ and $L^{\prime}$ are lattices, but in general $f$ needs not to be a morphism of lattices. However we have the following

Proposition 3.3. Let $L$ and $L^{\prime}$ be lattices.

(1) Let $f: L \rightarrow L^{\prime}$ be a morphism of join semi-lattices. If $L$ is finite, then $f$ considered as a morphism of posets has a right adjoint $f^{\dagger}: L^{\prime} \rightarrow L$ which is a morphism of meet semi-lattices.

(2) Let $f: L \rightarrow L^{\prime}$ be a morphism of meet semi-lattices. If $L^{\prime}$ is finite, then $f$ considered as a morphism of posets has a left adjoint $f^{*}: L^{\prime} \rightarrow L$ which is a morphism of join semi-lattices.

Proof. Take an element $y \in L^{\prime}$ and consider the set

$$
Q_{f, y}=\{x \in L \mid f x \leq y\}
$$

We claim that $Q_{f, y}$ is a join subsemilattice of $L$. First, the least element of $L$ belongs to $Q_{f, y}$ and next, if $x_{1}, x_{2} \in Q_{f, y}$, then $f\left(x_{i}\right) \leq y$ for $i=1,2$. Hence

$$
f\left(x_{1} \vee x_{2}\right)=f\left(x_{1}\right) \vee f\left(x_{2}\right) \leq y
$$

and $x_{1} \vee x_{2} \in Q_{f, y}$. By Lemma $3.2 Q_{f, y}$ has the greatest element. Hence we can use Proposition 3.1 to deduce existence of $f^{\dagger}$. Take now $y_{1}, y_{2} \in Y$. Then we have $\left(f x \leq y_{1} \wedge y_{2}\right) \Leftrightarrow\left(f(x) \leq y_{1}\right.$ and $\left.f(x) \leq y_{2}\right) \Leftrightarrow\left(x \leq f^{\dagger}\left(y_{1}\right)\right.$ and $\left.x \leq f^{\dagger}\left(y_{2}\right)\right) \Leftrightarrow$ $\left(x \leq f^{\dagger}\left(y_{1}\right) \wedge f^{\dagger}\left(y_{2}\right)\right)$. Hence

$$
f^{\dagger}\left(y_{1} \wedge y_{2}\right)=f^{\dagger}\left(y_{1}\right) \wedge f^{\dagger}\left(y_{2}\right)
$$

and the first assertion is proved. A similar argument shows the second assertion.

Corollary 3.4. The category of finite join semi-lattices is contravariantly equivalent to the category of finite meet semi-lattices. 
Proof. By Lemma 3.2 we can assume that both categories in question have finite lattices as objects. The duality functor from the category of finite join semi-lattices to the category of finite meet semi-lattices is the identity on objects and sends a join semi-lattice morphism $f$ into $f^{\dagger}$. The functor in the opposite direction is also identity on objects and sends a meet semi-lattice morphism $g$ into $g^{*}$.

3.2. Spec of finite semilattices. Lemma 2.3 reduces the study of $\operatorname{Spec}(M)$ to the case when $M$ is a join semilattice. Our aim is now to prove that for finite (=finitely generated) semilattice $L$ there exist a "natural" homeomorphism $\alpha_{L}: L \rightarrow \operatorname{Spec}(L)$, where $L$ is equipped with a topology, where open sets are exactly ideals of $L$.

Let $L$ be a join semilattice considered as a monoid. Take an element $a \in L$ and consider the set

$$
Q_{a}=\{x \in L \mid x \leq a\} .
$$

Let $\alpha(a)$ be the complement of $Q_{a}$ in $L$. Then we have

Lemma 3.5. (1) $Q_{a}$ is a subsemilattice.

(2) $(a \leq b) \Longleftrightarrow\left(Q_{a} \subset Q_{b}\right)$

(3) $\alpha(a) \in \operatorname{Spec}(L)$

(4) $\alpha_{L}: L \rightarrow \operatorname{Spec}(L)$ is an injective map.

(5) If $L$ is lattice, then $\alpha_{L}(a \wedge b)=\alpha_{L}(a) \cup \alpha_{L}(b)$.

Theorem 3.6. Let $L$ be a finite semilattice.

(1) For any prime ideal $\mathfrak{p}$ we let $\beta_{L}(\mathfrak{p})$ be the greatest element of the subsemilattice $L-\mathfrak{p}$, then for the map $\beta_{L}: \operatorname{Spec}(L) \rightarrow L$ one has $\beta_{L} \circ \alpha_{L}=i d$. Thus

$$
\alpha_{L}: L \rightarrow \operatorname{Spec}(L)
$$

is bijective.

(2) For a subset $S \subset L$, the set $\alpha_{L}(S)$ is an open subset of $\operatorname{Spec}(L)$ if and only if $S$ is an ideal.

(3) Let $f: L \rightarrow L^{\prime}$ be a morphism of finite join semilattices then one has the following commutative diagram

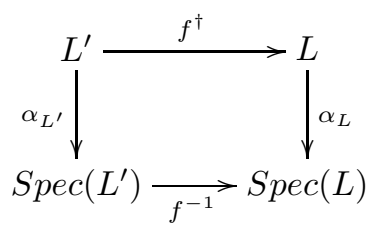

where as usual $f^{\dagger}$ denotes the right adjoint of $f$.

Proof. Take $a \in L$. Then we have

$$
\beta_{L} \circ \alpha_{L}(a)=\beta_{L}\left(L-Q_{a}\right)=\operatorname{Max}\left\{x \mid x \in Q_{a}\right\}=a
$$

and the first assertion follows. We also have

$$
\begin{aligned}
D(a)= & \{\mathfrak{p} \in \operatorname{Spec}(L) \mid a \notin \mathfrak{p}\}=\left\{\alpha_{L}(m) \mid a \notin \alpha_{L}(m)\right\}= \\
& \left\{\alpha_{L}(m) \mid a \in Q_{m}\right\}=\left\{\alpha_{L}(m) \mid m \in(a)\right\}
\end{aligned}
$$

Thus $\alpha^{-1}(D(a))=(a)$ and the second assertion also follows.

To see that the diagram commutes it suffice to prove that

$$
\beta_{L} \circ f^{-1} \circ \alpha_{L^{\prime}}=f^{\dagger}
$$

Take $y \in L^{\prime}$. Then $\beta_{L} \circ f^{-1} \circ \alpha_{L^{\prime}}(y)=\beta_{L} f^{-1}\left(L^{\prime}-Q_{y}\right)=\beta_{L}\left(L-f^{-1}\left(Q_{y}\right)\right)=$ $\operatorname{Max}\left\{x \in L \mid f(x) \in Q_{y}\right\}=\operatorname{Max}\{x \in L \mid f(x) \leq y\}=f^{\dagger}(y)$ and we are done. 
Corollary 3.7. (1) If $L$ is a finite semi-lattice then the composite

$$
L \stackrel{\alpha_{L}}{\longrightarrow} \operatorname{Spec}(L) \stackrel{\alpha_{\operatorname{Spec}(L)}}{\longrightarrow} \operatorname{Spec}(\operatorname{Spec}(L))
$$

is an isomorphism of semi-lattices, which is functorial in $L$.

(2) Any finite semi-lattice is the spectrum of a monoid.

Proof. The second part is a direct consequence of the first one. Since the bijection $\alpha_{L}$ reverses the ordering the first part follows.

Combine Theorem 3.6 and Theorem 2.3 to obtain the following result.

Corollary 3.8. (1) For any monoid $M$ there is an injective map

$$
\alpha_{M}: M^{s l} \rightarrow \operatorname{Spec}(M)
$$

which is bijective if $M$ is a finitely generated monoid.

(2) Let $M$ be a finitely generated monoid and let $f: M \rightarrow N$ be a morphism of monoids. Then one has the following commutative diagram

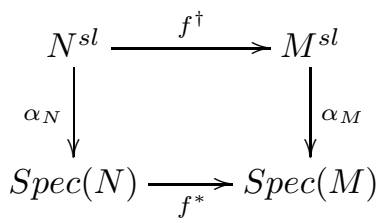

where as usual $f^{\dagger}$ is the right adjoint to the poset map $f_{*}: M^{s l} \rightarrow N^{s l}$.

(3) For any finitely generated monoid $M$ one has the natural bijection

$$
\operatorname{Spec}^{3}(M) \cong \operatorname{Spec}(M),
$$

where Spec ${ }^{3}$ is the three fold composite of Spec.

3.3. Infinite semilatices and not-finitely generated monoids. Asumume $L$ is a semilatice. We let $L_{\lambda}$ denote the collection of all finitely generated subsemilattices of $L$. Observe that each $L_{\lambda}$ is finite and they form a filtered system with respect to inclusion. If $f_{\lambda, \eta}: L_{\lambda} \subset L_{\eta}$ is an inclusion of finite subsemilattices, we have induced surjective morphism of dual meet-semilatices

$$
f_{\lambda, \eta}^{\dagger}: L_{\eta} \rightarrow L_{\lambda}
$$

In this way one obtains the inverse system of finite meet-semilattices. Let $L_{\infty}$ be the inverse limits of this system

Proposition 3.9. For any $L$ there is a bijection

$$
L_{\infty} \rightarrow \operatorname{Spec}(L)
$$

Proof. Since $L=\operatorname{colim} L_{\lambda}$ the result follows from Corollary 2.2 .

In this way we obtain a contravariant functor $L \mapsto L_{\infty}$ from the category of semilattices to the category of profine semilattices. For $L=M^{s l}$ we write $M_{\infty}^{\text {sl }}$ for $L_{\infty}$. Thus $M \rightarrow M_{\infty}^{s l}$ is a contavariant functor from monoids to the category of profinite semilatices. Combining the previous results we obtain a bijection

$$
\operatorname{Spec}(M) \rightarrow M_{\infty}^{s l}
$$

for any commutative monoid $M$. 


\section{REFERENCES}

[1] H. Brenner. Topologische und algebraische Filter. Dimplomarbeit. Ruhr-Universität Bocum. 1994. arXiv: 0302.235v1.

[2] A. Connes and C. Constani. Schemes over $\mathbb{F}_{1}$ and zeta functions. arXiv: 0903.2024v3.

[3] A. Connes and C. Constani. Characteristic 1, entropy and the absolute point. arXiv: $0911.3537 \mathrm{v} 1$.

[4] G. Cortiñas, C. Haesemeyer, M. E. Walker and C. Weibel. Toric varieties, monoid schemes and $c d h$ descent. arXiv:11061.389v1.

[5] C. Chu, O. Lorscheid and R. Santhanam. Sheaves and $K$-theory for $\mathbb{F}_{1}$-schemes. arXiv: $1010.2896 \mathrm{v} 2$.

[6] A. Deitmar. Schemes over F1. In: Number fields and function field. Two parallel worlds. Ed. by G. van der Geer, B. Moonen, R. Schoof. Progr. in Math. vol. 236, 2005.

[7] A. Deitmar. F1 Schemes and toric varieties. Contributions in Algebra and Geometry. 49(2008), 517-528.

[8] P. A. Grillet. Commutative semigroups. Kluwer Aacademic Publisher. 2001.

[9] K. Kato. Toric singularities. American Journal of Mathematics. 116(1994),1073-1099.

[10] J. López PEÑA and O. Lorscheid. Mapping F $_{1}$-land: An overview of geometries over the field with one element. arXiv: 0909.0069.

E-mail address: ilia_p@ymail.com 